\title{
A Comparative Study of Energy Consumption Sources for Wireless Sensor Networks
}

\author{
Mohammed AboZahhad, Mohammed Farrag, and Abdelhay Ali \\ Department of Electrical and Electronic Engineering, Assiut University, Assiut, \\ Egypt \\ E-mail:Zahhad@yahoo.com, \{Mohammed.farrag,Abdelhay\}@eng.au.edu.eg
}

\begin{abstract}
With the development of MEMS and CMOS technologies, the implementation of a huge number of wireless distributed micro-sensors that can be simply and rapidly deployed to form highly redundant, self-configuring, and ad hoc sensor networks. Sensor nodes are generally battery-devices, the critical facets to face concern are how to minimize energy consumption of nodes, so that the lifetime of sensing nodes can be maximized. The first step to achieve this goal is to know completely the sources of energy consumption in wireless sensor networks. In this paper, sources of energy consumption at various communication layers have been studied and investigated. Moreover, the energy consumption for the components of a typical sensor node and the impact of communication protocols stack on the energy consumption are discussed. In the sequel, the sources of energy consumption in each communication layer individually are studied. Then, a survey has been provided for existing energy models and the classification of these models into physical layer, MAC layer and cross-layer energy models.
\end{abstract}

Keywords: Wireless sensor networks, energy consumption, energy modeling, physical layer, cross-layer

\section{Introduction}

The combined and separated research advancement in the fields of computing, communication, and sensing has driven the research efforts in wireless sensor networks (WSNs) in recent years. Typically WSNs consists of interconnected sensor nodes, from a few to several thousand that are capable of not only sensing and computing, but also communicating with each other[1]. These networks are found usage in various areas, such as industrial process automation, environmental monitoring, and field surveillance[2].

Different from the traditional networks, the main characteristics of aWSNs are shortrange transmission, high sensor density, and low data rate. Depending on the application, there can also be stringent latency and bit error rate requirements. As a result of the random distributed nature and the large density of WSNs, battery replacement is a hard task. Actually, a major issue that prevents WSNs to be used in various application areas is the short battery life. Consequently, maximizing the battery lifetime of the sensor nodes is significant. The first step to achieve this goal is to know completely the sources of energy consumption in wireless sensor networks.

The functionality of a WSN node is generally executed through microelectronics of sensing unit, processing unit, communication unit and power unit [3]. Each unit has three conditions; active, idle and sleep. Energy consuming in each unit depends on the state of the unit. The communication system, used to transfer information between the sensor nodes, is a main block in every WSN system. Practical communication system design is aided by communication protocol stack. The communication protocol stack is a five-layer architecture, where each layer is responsible for specific sub-systems. The communication protocol stack is made up of a physical layer, data link layer, network layer, transport layer 
and application layer. Each layer has its own characteristics and has its effect on the energy consumed. The physical layer is responsible for frequency selection, carrier frequency generation, modulation, signal detection and data encryption. The function of the data link layer is how dose node communicates with other nodes, so it's related to retransmission due to collisions, overhearing. The network layer is responsible for choosing the route between source and destination. The transport layer helps to keep the flow of data if the application needs it. Application layer crossing point directly with the applicationand it makes common facilities for the application processes, and problems requests to the transport layer.

Cross-layer is targeted to give-and-take of information over layers to reach significant performance enhancement by developing the interaction between different protocol layers [4].

In this paper, the energy consumption of each hardware unit of a typical sensor node is discussed and some example unites are presented. Regarding to the communication protocols, the impact of communication protocols stacks of WSNs on energy consumption is discussed. Moreover, a survey of existing energy consumption models has been provided. These models have been classified into physical layer energy models, MAC layer energy models and cross-layer energy models.

The rest of this paper is organized as follows. Section 2 discusses judgment metrics on the WSNs. Section 3, is dedicated to the hardware of sensor node and energy consumed in each unit. Section 4, discuss the sources of energy consumption in each communication layer individually. Section 5, provide a survey of existing energy models in WSNs. In Section 6, we conclude the paper with a summary and give possible directions for the future work.

\section{Metrics in the WSNs}

There are several metrics that are used to judge the quality of WSN systems. Some of these include;

1) Network Lifetime (LT): which is a significant metric depending on several factors, including lifetime definition, energy consumption model, protocols, and network architecture, channel characteristics, and data collection initiation [5].

2) Energy Efficiency (EE): which aims to reduce the amount of energy consumption for a given task (e.g. Energy efficient clustering scheme (EECS))[6].

3) Energy-Per-Useful-Bit (EPUB): which captures overhead due to physical layer modulation [7].

4) Latency: which can be defined as the time required to send a packet through a network from source to the sink [8].

The network lifetime becomes a critical metric used to judge the quality of WSNs, where several techniques have been made to increase the network LT. Some of these include power aware storage, energy-aware MAC protocols, duty-cycling schemes, redundant placement of nodes, data dissemination and routing protocols, and tiered system architectures [9]. As we mentioned above that the network lifetime depends on the definition, so we listed below some common definitions of network lifetime which are used in previous work:

Definition 2.1: Network lifetime can be defined as the time duration from the beginning of the network job to the moment when the first nodedies [9].

Definition 2.2: Network lifetime is the maximum time after which the desired network performance cannot be achieved [10].

Definition 2.3: Network lifetime is defined as the time period during which the data rate is preserved above a minimum required datarate [11]. 
Definition 2.4: Network lifetime is defined as the time duration from start the first transmission in the WSNs to the moment when the number of nodes which have not finished their remaining energy falls below a threshold, which is depended on the type of application [5].

Definition 2.5: Network Lifetime can be defined as the time period, which the required Signal-to-Noise Ratio (SNR) at the sink is met with a specific probability [12].

\section{Hardware of Sensor Node}

The functionality of a sensing node is generally implemented through four basic units which are; transceiver, processing, sensing and power units as shown in Figure 1. The transceiver unit allows the sensor node to interconnect with other sensor nodes. The processing unit is used to allow management and data processing in the sensor node. The function of sensing unit is observing specific phenomenon. The power unit is used to provide the system supply voltage and it can be supported by an energy harvesting unit such as small-scale wind mills or solar cells. Also, it may have optional unit such as mobilize subsystem and location finding system[13-14]. Therefore, the average energy consumption per unit time of sensor node can be express as:

$$
E_{\text {hw }}=E_{\text {tran }}+E_{\text {comp }}+E_{\text {sen }}+E_{\text {pow }}
$$

where $E_{\text {tran }}, E_{\text {comp }}, E_{\text {sen }}$ and $E_{\text {pow }}$ are the energy consumed in communication subsystem, computing subsystem, Sensing subsystem and Power subsystem, respectively.

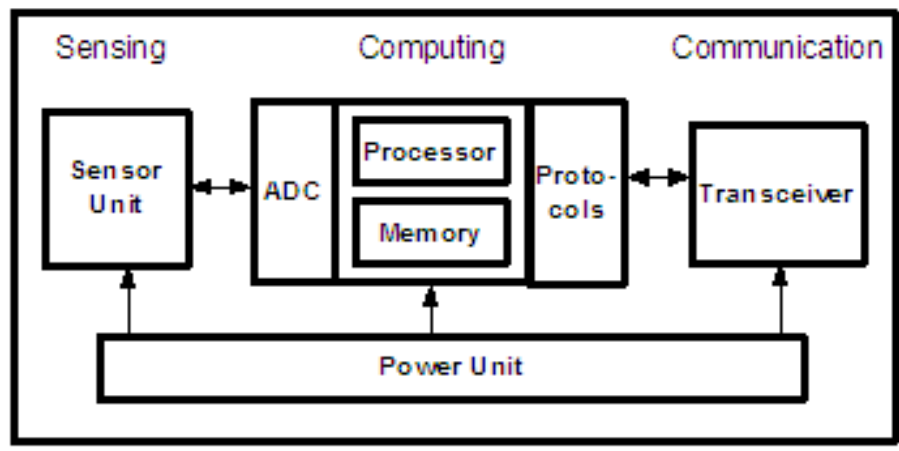

Figure 1. Sensor Node Hardware Architecture

\subsection{Communication Subsystem}

The Communication Subsystem is composed of transmitter circuit, receiver circuit, and an antenna which are used to send and receive packets. The purposes of the Communication Subsystem are the selection of a frequency channel, a transmit power, the modulation scheme, symbol synchronization, and clock generation.The energy consumption for a certain communication subsystem in sensor node can be expressed as [15]:

$$
\mathrm{E}_{\text {tran }}=\mathrm{P}_{\text {ont }} \mathrm{T}_{\text {on }}+\mathrm{P}_{\text {onr }} \mathrm{T}_{\text {on }}+\mathrm{P}_{\text {tr }} \mathrm{T}_{\text {tr }}+\mathrm{P}_{\mathrm{sm}} \mathrm{T}_{\text {sm }}+\mathrm{P}_{\mathrm{dc}} \mathrm{T}_{\text {on }}
$$

where the total time duration is divided between the communication active mode $T_{\text {on }}$, transient mode $\mathrm{T}_{\text {tr }}$ and sleep mode $\mathrm{T}_{\mathrm{sm}}$. During sleep mode, the power consumption $P_{s m}$ is dominated by the leaking current of switching transistors. This term is often neglected, i.e. $P_{s m}$ can be set to zero. The transient-mode time arises mainly from the frequency synthesizer settling time, and the settling time for other devices such as a mixer and power amplifier can be neglected and power consummation at thismode is $\mathrm{P}_{\mathrm{tr}}$. During active mode, power is consumed at digital 
circuits $\left(P_{d c}\right)$, analog circuits at the transmitter $\left(P_{\text {ont }}\right)$ and receiver side $\left(P_{\text {onr }}\right)$. The analogcircuit power terms can be expressed as

$$
\begin{gathered}
P_{\text {ont }}=P_{D c t}+P_{A c t}+P_{a m p}+P_{t}=P_{t o}+(\alpha+1) P_{t} \\
P_{\text {onr }}=P_{D c r}+P_{A c r}+P_{L N A}=P_{r o}
\end{gathered}
$$

where $P_{D c t}$ and $P_{A c t}$ are the power consumptions of the digital circuit at transmitter side and the analog circuit at transmitter side, respectively, the two components can be modeled as a constant, $P_{t o} . P_{a m p}$ and $P_{t}$ are the power consumptions of the power amplifier and transmitted power. $\alpha=\xi / \eta-1$ with $\xi$ and $\eta$ being the drain efficiency and the peak-to-average ratio, respectively. Similarly, $P_{D c r}, P_{A c r}$, and $P_{L N A}$ are the power consumptions of the digital circuit at receiver side, the analog circuit at receiver side, and LNA, respectively, the three components can be modeled as a constant, $P_{r o}$. The characteristics of some of low power transceiver units are summarized in Table 1 [16-18].

\begin{tabular}{|c|c|c|c|c|c|c|}
\hline Name & Manufacturer & $\begin{array}{l}\text { Band } \\
(\mathrm{MHz})\end{array}$ & $\begin{array}{c}\text { Data } \\
\text { rate } \\
(k b p s)\end{array}$ & $\begin{array}{l}\text { Sle } \\
\text { ep } \\
(\mu A \\
)\end{array}$ & $\begin{array}{l}\mathrm{T} \\
\mathrm{X} \\
(m \\
A)\end{array}$ & $\begin{array}{c}\mathrm{RX} \\
(m A)\end{array}$ \\
\hline MRF49XA & Microchip & $5^{433 / 868 / 91}$ & 256 & .3 & 15 & 11 \\
\hline MRF89XA & Microchip & 915 & 25 & .1 & 25 & 3 \\
\hline $\begin{array}{ll}\text { MCMRF24J4 } \\
0\end{array}$ & Microchip & 2400 & 250 & 2 & 22 & 18 \\
\hline AT86RF212B & Atmel & $0^{700 / 800 / 90}$ & 1000 & .2 & 18 & 9.2 \\
\hline NSnRF2401A & $\begin{array}{c}\text { Nordic } \\
\text { Semiconductor }\end{array}$ & 2400 & 1000 & 0.9 & $.0^{13}$ & 19.0 \\
\hline NSnRF24L01 & $\begin{array}{c}\text { Nordic } \\
\text { Semiconductor }\end{array}$ & 2400 & 2000 & 0.9 & $.3^{11}$ & 12.3 \\
\hline NSnRF905 & $\begin{array}{c}\text { Nordic } \\
\text { Semiconductor }\end{array}$ & $433-915$ & 50 & 2.5 & $.5^{12}$ & 14.0 \\
\hline nRF24L01+ & $\begin{array}{c}\text { Nordic } \\
\text { Semiconductor }\end{array}$ & 2400 & 250 & .9 & $.33^{11}$ & 13.5 \\
\hline RFMTR1001 & SRFMonolithic & 868 & 115.2 & 0.7 & 12 & 3.8 \\
\hline RFMTR3100 & SRFMonolithic & 433 & 576 & 0.7 & 10 & 7.0 \\
\hline SEXE1201A & Semtech & 433 & 64 & 0.2 & $.0{ }^{11}$ & 6.0 \\
\hline SEXE1203F & Semtech & $433-915$ & 152.3 & 0.2 & $.0^{33}$ & 14.0 \\
\hline TICC2420 & Texas Instruments & 2400 & 250 & 1 & $.4{ }^{17}$ & 18.8 \\
\hline TICC2500 & Texas Instruments & 2400 & 500 & 0.4 & $.2^{21}$ & 17.0 \\
\hline TICC1000 & Texas Instruments & $433-915$ & 76.8 & 0.2 & $.4{ }^{10}$ & 9.3 \\
\hline TICC1100 & Texas Instruments & $433-915$ & 500 & 0.4 & $.55^{15}$ & 16.5 \\
\hline TICC2538 & Texas Instruments & 2400 & 250 & 1.3 & 24 & 20 \\
\hline TICC2531 & Texas Instruments & 2400 & 250 & .4 & 29 & 20.5 \\
\hline
\end{tabular}

Table 1.Radio Features, Current Consumptions, and Energy Efficiencies 


\subsection{Computing Subsystem}

The central component of sensor node is Computing Subsystem which is used to allow management and data processing in the sensor node. Micro-Controller Unit (MCU)is usually implements the Computing Subsystem, which is consist of a microprocessor, memories, Analog-to-Digital Converter (ADC), configurable I/O ports, timers, and other peripherals[16-19]. Energy consumed in a computing subsystem can be expressed as

$$
E_{\text {comp }}=E_{\text {pro }}+E_{\text {logg }}
$$

Where $E_{\text {pro }}$ is the total energy dissipation by MCU used for data processing/aggregation b bit packet and $E_{l o g g}$ is consumes energy used for reading b bit packet data and writing it into memory. $E_{\text {pro }}$ and $E_{\text {logg }}$ can be expressed as [11], [23] :

$$
\begin{gathered}
E_{\text {pro }}=b N_{c y c} C_{\text {avg }} V_{\text {sup }}^{2}+b V_{\text {sup }}\left(I_{o} e^{\frac{V_{\text {sup }}}{n_{p} V_{t}}}\right)\left(\frac{N_{c y c}}{f}\right) \\
E_{\text {logg }}=E_{\text {write }}+E_{\text {read }}=\frac{b V_{\text {sup }}}{8}\left(I_{\text {write }} T_{\text {write }}+I_{\text {read }} T_{\text {read }}\right)
\end{gathered}
$$

Where $V_{\text {sup }}$ is the supply voltage, $N_{c y c}$ is the number of clock cycles per task, $C_{a v g}$ is the average capacitance switched per cycle, $I_{o}$ is the leakage current, $n_{p}$ is the constant which depends on the processor, $V_{t}$ is the thermal voltage, $f$ is sensor frequency, $E_{\text {write }}$ is energy consumption for writing data, $E_{\text {read }}$ is energy consumption for reading b bit packet data, $I_{\text {write }}$ and $I_{\text {read }}$ are current for writing and reading 1 byte data, and $T_{\text {write }}$ and $T_{\text {read }}$ are Time for writing and reading 1 byte data. The Features of MCUs from several manufacturers are given in Table 2 [16-19].

\subsection{Sensing Subsystem}

There are wide ranges of low power sensors appropriate for WSNs. Small sensing time, acceptable accuracy and low power consumption are essential requirements for sensing. The energy consumption per unit time in sensing unit at sensor node can be express as [20].

$$
E_{\text {Sensing }}=\frac{T_{\text {sense }}}{T} b V_{\text {sub }} I_{\text {sense }}
$$

where $I_{\text {sense }}$ is the total current required for sensing activity, $T_{\text {sense }}$ is the sensing time, $T$ is the sensing period, and $b$ is the number of bit which generate at each activity. The features of some low power sensors are given in Table 3[16-21]

Table2.The Comparison of Features of Low Power MCUs

\begin{tabular}{|l|l|c|c|c|c|}
\hline \multicolumn{1}{|c|}{ Name } & Manufacturer & $\begin{array}{r}\text { SRA } \\
\mathbf{M}(\mathbf{k B})\end{array}$ & $\begin{array}{c}\text { FLAS } \\
\mathbf{H}(\mathbf{k B})\end{array}$ & $\begin{array}{c}\text { 1MIPS } \\
(\mathbf{m A})\end{array}$ & $\begin{array}{c}\text { Sleep } \\
(\boldsymbol{\mu A})\end{array}$ \\
\hline AT89C51RE2 (8051) & Atmel & 8 & 128 & 7.4 & 75 \\
\hline ATmega103L (AVR) & Atmel & 4 & 128 & 1.38 & 1 \\
\hline CY8C29666 & Cypress & 2 & 32 & 10 & 5 \\
\hline M68HC08 & Freescale & 2 & 61 & 3.75 & 22 \\
\hline PIC18LF8722 & Microchip & 3.9 & 128 & 1.0 & 2.32 \\
\hline PIC24FJ128 & Microchip & 8 & 128 & 1.6 & 21 \\
\hline XE8802 (CoolRisc) & Semtech & 1 & 22 & 0.3 & 1.9 \\
\hline MSP430F1611 & Texas & 10 & 48 & 0.33 & 1.3 \\
\hline
\end{tabular}


Table 3. Features of Typical Sensors

\begin{tabular}{|c|c|c|c|}
\hline Name & Type & $\begin{array}{l}\begin{array}{l}\text { Sensing } \\
\text { time }\end{array} \\
\end{array}$ & $\begin{array}{r}\text { Active } \\
\text { current }\end{array}$ \\
\hline SLG64-0075 & Liquid-Flow & $30 \mathrm{~ms}$ & $5.1 \mathrm{~mA}$ \\
\hline SFM4100 & Gases- Flow & $4.6 \mathrm{~ms}$ & $12.5 \mathrm{~mA}$ \\
\hline VTISCA3000 & Acceleration & $10 \mathrm{~ms}$ & $120 \mu \mathrm{A}$ \\
\hline VTISCP1000 & Airpressure & $110 \mathrm{~ms}$ & $25 \mu \mathrm{A}$ \\
\hline 5 SensorionSHT1 & Humidity & $210 \mathrm{~ms}$ & $300 \mu \mathrm{A}$ \\
\hline $\begin{array}{l}\text { AvagoAPDS- } \\
9002\end{array}$ & Illumination & $1.0 \mathrm{~ms}$ & $2.0 \mathrm{~mA}$ \\
\hline HitachiHM55B & Magneticfield & $30 \mathrm{~ms}$ & $9.0 \mathrm{~mA}$ \\
\hline 3 FastraxiTRAX0 & Position & $4.0 \mathrm{~s}$ & $32 \mathrm{~mA}$ \\
\hline DallasDS620U & Temperature & $200 \mathrm{~ms}$ & $800 \mu \mathrm{A}$ \\
\hline
\end{tabular}

\subsection{Power Subsystem}

The power subsystem stores energy in battery and supply suitable voltage levels. It consists of battery and a voltage regulator[22]. The energy consumed in the power subsystem can be express as

$$
E_{\text {pow }}=\left(V_{\text {in }}-V_{\text {out }}\right) \times I_{\text {out }} \times T
$$

Where $V_{\text {in }}$ and,$V_{\text {out }}$ are the input voltage and output voltage respectively, $\mathrm{I}_{\text {out }}$ output Current and $\mathrm{T}$ is Operate time.

Some time, harvesting units are appended to power subsystem. Due to nature and environment, energy harvesting units don't provide a constant power rate. So, these units are mainly worked in combination with a storage device such as a rechargeable battery or super-capacitor. Storage device stores additional energy and offers it later, when there is no energy can be harvested from the environment. We listed below some common scavenging sources which are used in WSNs[23] and the typical amount of power made by diverse energy sources is summarized in Table 4 [19].

- Solar cells use light of sun to produce electricity and it are very common energy harvesting sources. Small panel supplies sufficient power to sensor node. A rechargeable battery or a super-capacitor is usually used with solar cells.

- Small- scale wind mills are unusually energy harvesting sources and it appropriate for outdoor applications.

- Radiant energy is harvesting technique that convert energy from electromagnetic waves.

- Vibration energy is harvesting method that convert vibration energy. It common uses with civil engineering applications.

- Thermal energy is harvesting technique that convert the temperature difference between two materials to energy. here are a wide

Table 4. Typical Amount of Power Made by Diverse Energy Scavenging

\begin{tabular}{|l|c|l|}
\hline \multicolumn{1}{|c|}{ Energy Source } & Duration & \multicolumn{1}{|c|}{ Power Density } \\
\hline Temperature Difference & $40 \mu W / \mathrm{cm}^{3} / 5^{\circ} \mathrm{C}$ & Continuous \\
\hline Piezoelectric & $200 \mu \mathrm{W} / \mathrm{cm}^{3}$ & Operation (e.g button push) \\
\hline Air Flow & $380 \mu \mathrm{W} / \mathrm{cm}^{3} / 5 \mathrm{~m} / \mathrm{s}$ & Continuous \\
\hline Solar cell & $15 \mathrm{~mW} / \mathrm{cm}^{2}$ & Continuous \\
\hline
\end{tabular}




\section{Communication Protocols Stack}

In WSNs, there are five communication layers: physical layer, data link layer, network layer, transport layer, and application layer as shown in Figure 2[24]. Each layer in the communication protocol stack has its own parameters which effect on the energy consumption in this layer as shown in Figure 3. In the following, we discuss the most relevant features of each communication layer and its impact on energy consumption.

\subsection{Physical Layer}

Physical layer is the lower layer of the communication layers and it provides the interface to the sensor node to communicate with other node over the physical medium. The function of physical layer is carrier frequency generation, modulation, and signal detection. In WSNs, the physical layer should be given a special care due to the characteristic constraints, such as low-power consumption and hardware design , such as low-power consumption and hardware design [24-25].

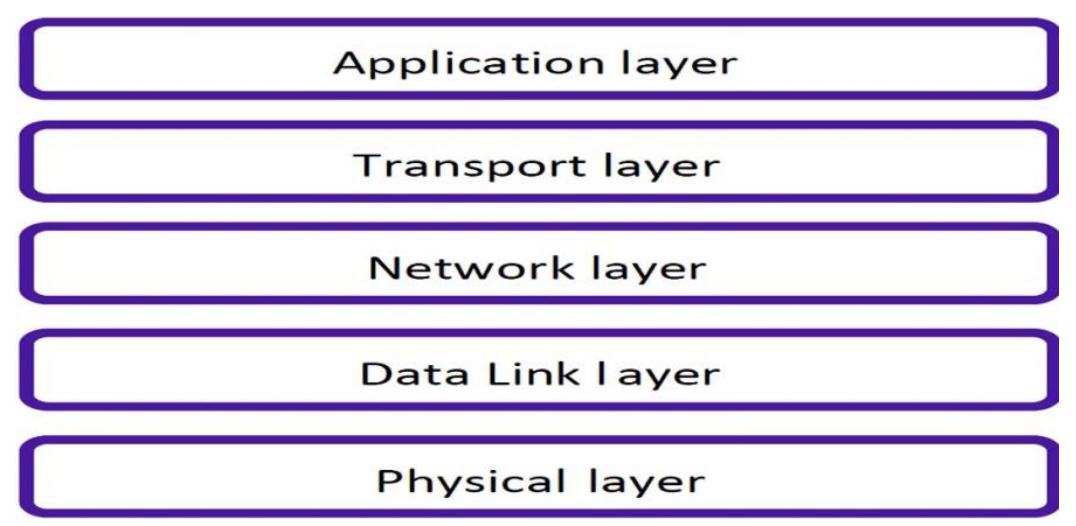

Figure 2. Architecture of a WSN Communication Protocol

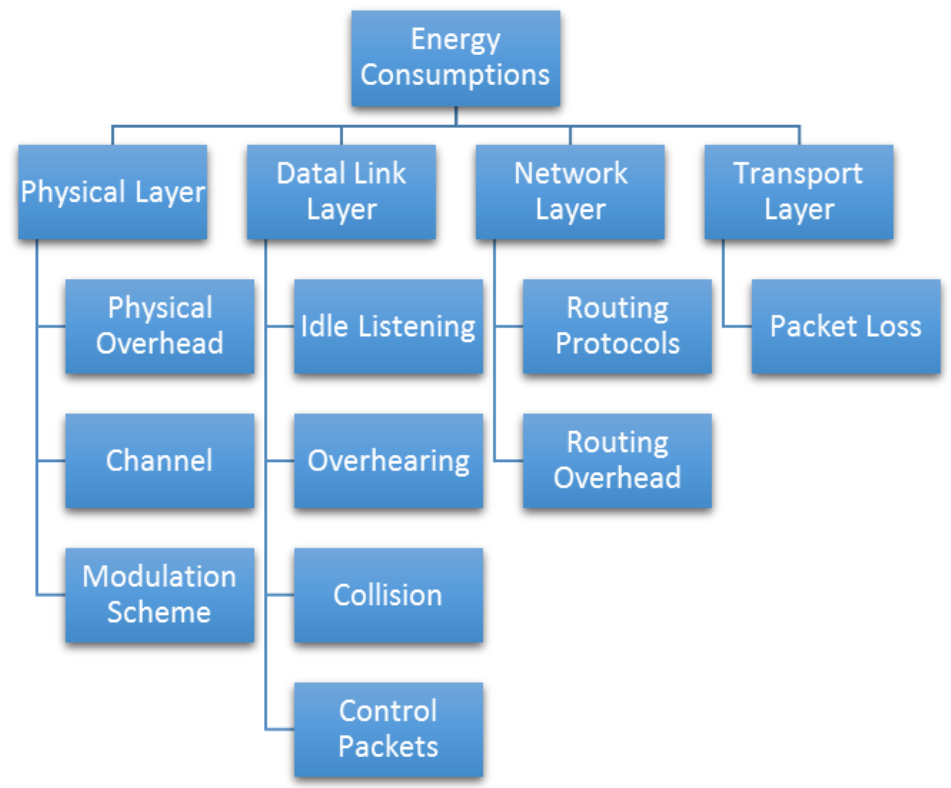

Figure 3. Energy consumption parameters in WSNs

In physical layer, processing unit has two low-Power techniques for saving energy; DVS (Dynamic Voltage Scaling) and DMS (Dynamic Modulation Scaling. DVS technique based on varying computation load with time, and highest system performance 
is not continually necessary. Actually, this technique is used for reducing CPU energy consumption by dynamically changing the supply voltage and clock speed based on the instantaneous amount of work. Similarly in DMS, the modulation level can be adjust in accord with the instantaneous communication load [4].

\subsection{Data link Layer}

The second layer of the communication stack is Data link layer (DLL) and it generally consists of two sub-layers: Logical Link Control (LLC) and Medium Access Control (MAC). The functions of MAC protocols are providing connections for the routing protocol and managing transceiver unit on a shared wireless medium. In general, there are two categories of MAC protocols [25].

1) Schedule-based Protocols which is depend on scheduling and reservation. Time-division multiple access (TDMA) is a good example of these type of protocols, in which the time is divided into several time slots. Each time slot is assigned to a sensor node and a node can transfer message during its assigned time.

2) Contention-Based MAC Protocols, in which nodes compete to reserve shared wireless media and trying to avoid packet collisions. In this type of MAC protocols, duty cycling is used to advanced energy saving, where the time is divided into a long sleep period and a small active period. This type of MAC protocols can be classified, according to the synchronization of data exchanged, into two categories: unsynchronized and synchronized protocols [26, 27].

MAC protocols have a very high effect on network performance and energy consumption. Therefore, a MAC protocol should minimize [19]:

- Idle listening: Idle listening happens when a node is actively receiving a channel, but there is no significative action on the channel resulting loss of energy[28].

- Collisions: Collisions occurs when two or more nodes transmit simultaneously at the same channel Thus, the received packet is presumably corrupted producing unusable receive fee at the destination node and unusable transmit fee at the source node.

- Overhearing: Overhearing occurs when node receives packets that are sent to the shared medium and they are not destined for it and consumes unnecessarily energy.

- Protocol overhead: In WSN system, short packets, protocol headers and trailers are used, which causing significant energy consumption. Moreover, the energy consumption due to control packet exchange, such as ACK packet may be very significant.

Therefore, energy consumed in the sensor node with take in to account MAC layer can express as

$$
E_{\text {mac }}=E_{h w}+E_{\text {ohear }}+E_{\text {ohead }}+E_{\text {retx }}+E_{\text {idle }}
$$

where $E_{\text {ohear }}$ and $E_{\text {ohead }}$ are the energy consumed due to overhearing and overhead, respectively, $E_{\text {retx }}$ is the energy consumed due to retransmitted losses packets, which is resulting from collisions, and $E_{\text {idle }}$ is wasted energy in idle state.

\subsection{Network Layer}

The third layer in the WSNs communication protocol stack is the network layer. The functions of network layer are network routing, error control, source to destination packet delivery, network segmentation and de-segmentation, and flow control. In WSNs, finding the route between a source node and destination node is task of the routing protocols. Based on the operation of WSNs routing protocols, it can be categorized into: nodecentric, data-centric, location based, multipath routing, hierarchical protocols, mobilitybased protocols, and QoS-based protocols [29, 30]. At the network layer, routing 
protocols have a very high effect on network performance and energy consumption. Thus, it had better take into account the following goals in to decreasethe energy consumption and extend lifetime [25].

- Minimization of the Energy Consumed per bit or Packet.

The energy consumed per bit or Packet is the energy that is used for the transmission of the bit/Packet from the source to the sink.

\section{- Maximization of the Network lifetime.}

The network lifetime is the duration time before the network loses its connectivity.

- Minimization of the Budget per Packet and Node.

The budget per packet is defined as the energy consumed for transfer a packet from the source node to the sink node over the shortest route. The objective is to reduce the budget per packet, which can be reached by avoiding those sensor nodes with the smallestremaining energy on selected routes. Also, the budget per node is defined as the amount of energy consumed by each senor node for transferring a packet to the sink node. This objective is to reduce the budget per node with the aim of the lifetime is maximized.

\subsection{Transport layer}

The fourth layer in the WSN communication protocol stack is the transport layer. It provides services such as flow control, multiplexing and reliability between end users. The purpose of this layer is to provide reliable data transfer between source and destination, thus relieving the upper layers' responsibility for providing reliable and cost-effective data transfer [22].

In the context of WSNs, not much work has been done to address the energy consumption issue at the transport layer. But it is easy to find out its effect on the energy consumed. Where it is at calculating the energy consumed in sensor node a new term $E_{\text {retrspos }}$ appears in the equation as a result of re-transmitter in Transport layer.

\subsection{Application layer}

The last layer of the WSN communication protocol stack is the application layer and it makes common services for the application. The Sensor Management Protocol (SMP), and the Sensor Query and Data Dissemination Protocol (SQDDP) are examples of application protocols in WSNs [3], [16].

\section{Energy Consumption Models}

The energy consumption model may be defined as designing and analyzing a mathematical representation of a WSN to study the effect of changing the system parameters. The behavior of energy consumption model is a function of its parameters. The parameters may be set when design time, in which case they may be considered as fixed resources (e.g., Initial energy), or they may change after a system has been implemented (e.g., Packet sizes and transmitted power).

There are several previous attempts to model energy consumption for sensor node. In general energy consumption models can be classified into three categories, physical layer energy models, MAC layer energy models and cross-layer energy models. Physical layer energy models are models that take into account the parameters of energy consumption at physical layer only .This include transmitted power, received power and sensing power. MAC layer energy models are models that take into account the parameters of energy consumption at physical layer and parameters of energy consumption at MAC layer .This include overhead of MAC protocol, overhearing and collision. Cross-layer energy models 
are models that take into account parameters of energy consumption at various communication layers [31]. We summarized energy consumption parameters that are considered by various energy models in Table [5].

Table 5. Energy Consumption Parameters Considered in Various Energy Models

\begin{tabular}{|c|c|c|c|c|c|c|}
\hline & $\begin{array}{l}\text { Transmit } \\
\text { ted power }\end{array}$ & $\begin{array}{l}\text { TX circuit } \\
\text { power }\end{array}$ & $\begin{array}{r}\mathrm{RX} \\
\text { circuit } \\
\text { Power }\end{array}$ & $\begin{array}{l}\text { Processi } \\
\text { ng power }\end{array}$ & $\begin{array}{l}\text { Sensin } \\
\text { g power }\end{array}$ & Overhead \\
\hline [32] & $\sqrt{ }$ & - & $\sqrt{ }$ & - & - & - \\
\hline [33] & $\sqrt{ }$ & - & $\sqrt{ }$ & - & - & - \\
\hline [34] & $\sqrt{ }$ & - & $\sqrt{ }$ & - & - & - \\
\hline [7] & $\sqrt{ }$ & - & $\sqrt{ }$ & - & - & $\sqrt{ }$ \\
\hline [35] & $\sqrt{ }$ & $\sqrt{ }$ & $\sqrt{ }$ & $\sqrt{ }$ & - & - \\
\hline [36] & $\sqrt{ }$ & $\sqrt{ }$ & $\sqrt{ }$ & - & - & - \\
\hline [37] & $\sqrt{ }$ & $\sqrt{ }$ & $\sqrt{ }$ & - & - & - \\
\hline [38] & $\sqrt{ }$ & - & $\sqrt{ }$ & - & $\sqrt{ }$ & - \\
\hline [39] & $\sqrt{ }$ & $\sqrt{ }$ & $\sqrt{ }$ & - & $\sqrt{ }$ & - \\
\hline [40] & $\sqrt{ }$ & - & $\sqrt{ }$ & - & $\sqrt{ }$ & - \\
\hline
\end{tabular}

\section{Conclusions and Future Work}

Energy consumption in WSNs is extremely important due to limitation of power sources. In this paper, an overview of WSNs constrains and judgment metrics such as lifetime and latency are given. Moreover, we have given an introduction to source of energy consumption at each layer. Then, surveys have been provided for existing energy models. These models are classified into physical layer, MAC layer and cross-layer energy models. However, as the energy-awareness must be incorporated into all aspects of system hierarchy, there are still many open issues for future research. Future work will include, providing a new energy model which capture all energy consumption sources in all communication layers stack.

\section{References}

[1] I. F. Akyildiz, W. Su, Y. Sankarasubramaniam, and E. Cayirci, "Wireless sensor networks, a survey," Comput. networks, vol. 38, no. 4, pp. 393-422, (2002).

[2] J. Yick, B. Mukherjee, and D. Ghosal, "Wireless sensor network survey," Comput. Networks, vol. 52 , no. 12, pp. 2292-2330, (2008) August.

[3] M. Abo-zahhad, O. Amin, M. Farrag, and A. Ali, "A Survey on Protocols , Platforms and Simulation Tools for Wireless Sensor Networks,” Int. J. Energy, Inf. Commun., vol. 5, no. 6, pp. 17-34, (2014).

[4] S. Misra, S. ChandraMisra and I. Woungang, "Guide toWireless Mesh Networks. Springer, (2009).

[5] Y. Chen and Q. Zhao, "On the lifetime of wireless sensor networks," IEEE Commun. Lett., vol. 9, no. 11, pp. 976-978, (2005).

[6] M. Ye, C. Li, G. Chen, and J. Wu, "An Energy Efficient Clustering Scheme in Wireless Sensor Networks," Science (80-. )., vol. 3, pp. 99-119, (2007).

[7] J. Ammer and J. Rabaey, "The Energy-per-Useful-Bit Metric for Evaluating and Optimizing Sensor Network Physical Layers," Sens. Ad Hoc Commun. Networks, 2006. SECON'06. 2006 3rd Annu. IEEE Commun. Soc., vol. 2, pp. 1-6, (2006).

[8] Y. Wang, M. C. Vuran, and S. Goddard, "Cross-Layer Analysis of the End-to-End Delay Distribution in Wireless Sensor Networks,” IEEE/ACM Trans. Netw., vol. 20, no. 1, pp. 305-318, (2012)February.

[9] S. Ehsan, B. Hamdaoui and M. Guizani, "Radio and Medium Access Contention Aware Routing for Lifetime Maximization in Multichannel Sensor Networks," IEEE Trans. Wirel. Commun., vol. 11, no. 9, pp. 3058-3067, (2012)Septmber.

[10] H. Jaleel, S. Member and A. Rahmani, "Probabilistic Lifetime Maximization of Sensor Networks," IEEE Trans. Automat. Contr., vol. 58, no. 2, (2013).

[11] E. P. Allocation, M. Hajiaghayi, S. Member, M. Dong, S. Member, and B. Liang, "Maximizing Lifetime in Relay Cooperation Through," IEEE Trans. Signal Process., vol. 58, no. 8, pp. 4354-4366, (2010).

[12] W. J. Huang, Y. W. P. Hong, and C. C. Jay Kuo, "Lifetime maximization for amplify-and-forward cooperative networks,” IEEE Trans. Wirel. Commun., vol. 7, no. 5, pp. 1800-1805, (2008). 
[13] M. Abo-zahhad, M. Farrag, A. Ali and O. Amin, "Energy Consumption and Lifetime Analysis for Wireless Sensor Networks," in 32nd National Radio Science Conference (NRSC), (2015), Egypt.

[14] H. Zhou, D. Luo, Y. Gao and D. Zuo, "Modeling of Node Energy Consumption for Wireless Sensor Networks," vol. 2011, (2011) January, pp. 18-23,.

[15] O. Amin, S. Bavarian and L. Lampe, "Cooperative Techniques for Energy-Efficient Wireless Communications," in Green Radio Communication Networks, (2012), pp. 125-151.

[16] M. Kohvakka, "Medium Access Control and Hardware Prototype Designs for Low-Energy Wireless Sensor Networks," Tampereen teknillinen yliopisto. Julkaisu-Tampere University of Technology. Publication, vol. 808, (2009).

[17] "Microchip Corporation.” [Online]. Available: http://www.microchip.com/.

[18] "RF Transceiver." [Online]. Available: http://www.nordicsemi.com/.

[19] J. Suhonen, M. Kohvakka, V. Kaseva, T. D. Hämäläinen and M. Hännikäinen, "Low-power Wireless Sensor Networks", Protocols, Services and Applications, (2012), Springer.

[20] M. N. Halgamuge, M. Zukerman, and K. Ramamohanarao, "An Estimation of Sensor Energy Consumption,” Prog. Electromagn. Res. B, vol. 12, pp. 259-295, (2009).

[21] "Sensirion AG." [Online]. Available: http://www.sensirion.com.

[22] A. Belen, G. Hernando, J. Fernan, M. Ortega, J. Manuel, L. Navarro, A. Prayati and L. Redondo-Lopez, "Problem Solving for Wireless Sensor Networks", (2008), Springer-Verlag London.

[23] R. Bischoff, J. Meyer, and G. Feltrin, "Wireless Sensor Network Platforms," Encycl. Struct. Heal. Monit., pp. 1-10, (2009).

[24] A. Abed, A. Alkhatib and G. S. Baicher, "Wireless Sensor Network Architecture," in International Conference on Computer Networks and Communication Systems, vol. 35, no. Cncs,(2012), pp. 11-15.

[25] J. Zheng and A. Jamalipour, "WIreless Sensor Networks", A Networking Prespective. John Wiley \& Sons, (2009).

[26] P. Huang, L. Xiao, S. Member, S. Soltani, S. Member, M. W. Mutka and N. Xi, "The Evolution of MAC Protocols in Wireless Sensor Networks, A Survey," IEEE Commun. Surv. Tutorials, vol. 15, no. 1,(2013), pp. 101-120.

[27] K. Nguyen and Y. Ji, "Asynchronous MAC Protocol with QoS Awareness in Wireless Sensor Networks," in Globecom (2012)Ad Hoc and Sensor Networking Symposium Asynchronous, (2012), pp. 573-577.

[28] M. Abo-zahhad, M. Farrag, A. Ali and O. Amin, "An Energy Consumption Model for Wireless Sensor Networks," in 2015 5th Annual International Conference on Energy Aware Computing Systems and Applications (ICEAC), (2015).

[29] S. K. Singh, M. P. Singh, and D. K. Sing, "Routing Protocols in Wireless Sensor Networks," Int. J. Comput. Sci. Eng. Surv., vol. 1, no. 2, pp. 63-83, (2010).

[30] S. Kumar, A. Kumar and R. k. Vishwkarma, "A Survey on Routing Protocol for Wireless Sensor Network,” Int. J. Adv. Res. Comput. Eng. Technol., vol. 2, no. 2, pp. 750-755, (2013).

[31] M. A. zahhad, O. Amin, M. Farrag and A. Ali, "Survey on Energy Consumption Models in Wireless Sensor Networks," Open Trans. Wirel. Sens. Netw., vol. 1, no. 1, (2014).

[32] S. He, J. Chen, S. Member, D. K. Y. Yau and Y. Sun, "Cross Layer Optimization of Correlated Data Gathering in Wireless Sensor Networks," Mob. Comput. IEEE Trans., vol. 11, no. 11, pp. 1678-1691, (2012).

[33] J. H. Chang and L. Tassiulas, "Maximum Lifetime Routing in Wireless Sensor Networks," IEEE/ACM Trans. Netw., vol. 12, no. 4, pp. 609-619, (2004)August.

[34] Y. Gu, I. C. Society and Y. Ji, "ESWC, Efficient Scheduling for the Mobile Sink in Wireless Sensor Networks with Delay Constraint”,IEEE Trans. PARALLEL Distrib. Syst., vol. 24, no. 7, pp. 13101320, (2013).

[35] T. Melodia, S. Member and D. Pompili, "Communication and Coordination in Wireless Sensor and Actor Networks,” IEEE Trans. Mob. Comput., vol. 6, no. 10, pp. 1116-1129, (2007).

[36] D. Tudose, L. Gheorghe and N. Tăpuş, "Radio transceiver consumption modeling for multi-hop wireless sensor networks," UPB Sci. Bull. Ser. C Electr. Eng., vol. 75, no. 1, pp. 17-26, (2013).

[37] Q. Wang, M. Hempstead and W. Yang, "A Realistic Power Consumption Model for Wireless Sensor Network Devices,” 2006 3rd Annu. IEEE Commun. Soc. Sens. Ad Hoc Commun. Networks, pp. 286295, (2006).

[38] M. Cheng, X. Gong and L. Cai, "Joint routing and link rate allocation under bandwidth and energy constraints in sensor networks," IEEE Trans. Wirel. Commun., vol. 8, no. 7, pp. 3770-3779, (2009) July.

[39] J. Riku, "Joint Data Rate and Power Allocation for Lifetime Maximization in Interference Limited Ad Hoc Networks," IEEE Trans. Wirel. Commun., vol. 5, no. 5, pp. 1086-1094, (2006).

[40] M. Zheng, H. Yu, J. Zheng and P. Zeng, "Tradeoff between utility and lifetime in energy-constrained wireless sensor networks," J. Control Theory Appl., vol. 8, no. 1, pp. 75-80, (2010)January. 


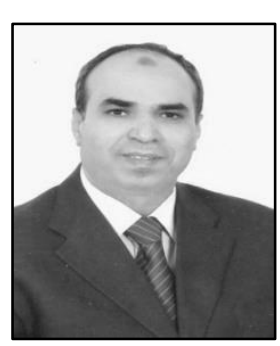

\section{Authors}

Prof. Mohammed Abo-Zahhad, He received his B.S.E.E. and M.S.E.E degrees in electrical engineering in 1979 and 1983 respectively, both from Assiut University (AU), Egypt. In 1988, he received $\mathrm{Ph}$. D. degree from the University of Kent at Canterbury, UK and AU (channel system). His research interests include switched-capacitor, optical and digital filters, biomedical and genomic signal processing, speech processing, data compression, wavelet-transforms, genetic algorithms, immune algorithms, wireless sensor networks and electronic systems. He has published more than 115 papers in national and international journals and conferences in the above fields. Professor AboZahhad is currently a Professor of Electronics and Communication Engineering, since Jan. 1999. Also, he is the director of AU Management Information System (MIS) center and a vice-dean for graduated studies, Faculty of Engineering, AU, since August 2006. From August 2006 till July 2013 he was the vice dean for graduate studies, Faculty of Engineering, AU. Since November 2013 he has been elected as the chair of Electrical and electronics engineering Department, AU. He is a member of the European Society of Circuit Theory and Applications, since 1998 and a senior IEEE member, since 2000.

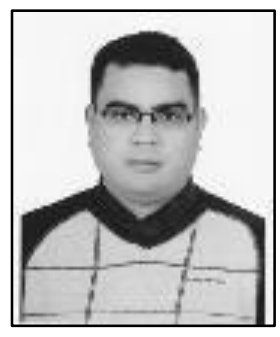

Dr. Mohammed Farrag, He received his B.Sc. degree in electrical and electronics engineering from Assiut University, Assiut, Egypt, in 2001, the M.Sc. degree in electrical engineering from Assiut University, Assiut, Egypt, in 2008, and the Ph.D. degree in electrical and electronics from Egypt-Japan University of Science and Technology, in 2013. His research interests include cooperative communications, Dynamic spectrum access, Cognitive Radio, orthogonal frequency-division multiplexing, green communication, and Compressive sensing applications.

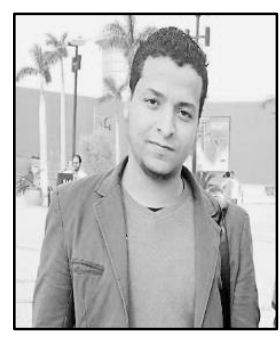

Eng. Abdelhay Ali, He received his B.Sc. (honors) degree in Electrical and Electronics Engineering Department, Faculty of Engineering, Assiut University, Assiut, Egypt, in 2012. He is currently a teaching assistantat electrical engineering department, Assiut University, Egypt. He pursues the M.S. degree in modeling of wireless sensor networks with maximum lifetime and minimum energy consumption. His main research interest is in wireless sensor networks with specific focus on maximize lifetime of wireless sensor networks. 